\title{
Diagnóstico de necesidades educativas para la construcción de programas de educación física en Educación Media Superior del Estado de México
}

\section{Diagnosis of educational needs for the construction of physical education programs in Upper Middle Education of the Estado de Mexico}

Claudia Raquel Peimbert Angulo ${ }^{1}$

Celso Castaño Enríquez ${ }^{2}$

Héctor Meza Espinoza ${ }^{3}$

\section{Resumen}

El problema que se plantea en este documento es la ausencia de programas de educación Física para el nivel Medio Superior en el Estado de México. Ante esta circunstancia, surge la necesidad de construcción de un diagnóstico que reflejara la realidad educativa. Se presentan resultados y análisis de encuestas aplicadas a docentes y alumnos de Educación Media Superior del Estado de México. La información obtenida sirvió de fundamento para elaborar un diagnóstico educativo de necesidades y expectativas que determinaron la pertinencia y factibilidad de programas para la asignatura de Educación Física, que han de implementarse en el ciclo escolar 2018-2019.

1 Claudia Raquel Peimbert Angulo. Docente de la Escuela Normal de Educación Física Ignacio M. Beteta, Estado de México. Es Maestra en Comunicación y Tecnología Educativa. Correo electrónico: raquelpeimbert@gmail.com

ID: http://orcid.org/0000-0002-8090-6309

2 Celso Castaño Enríquez. Docente de la Escuela Normal de Educación Física Ignacio M. Beteta, Estado de México. Maestro en Ciencias de la Educación y jefe del Departamento de Posgrado de la institución. Correo electrónico: ce casten@yahoo.com.mx

ID: http://orcid.org/0000-0003-0536-0489

${ }^{3}$ Héctor Meza Espinoza. Docente de la Escuela Normal de Educación Física Ignacio M. Beteta, Estado de México. Maestro en Desarrollo de la Motricidad y miembro del Departamento de Posgrado de la institución. Correo electrónico: hec mez es@yahoo.com.mx ID: http://orcid.org/0000-0003-3768-6440 
RECIE. Revista Electrónica Científica de Investigación Educativa Vol. 4, núm. 2, enero-diciembre 2019, pp. 1049-1057.

\title{
Palabras clave
}

Educación física, diagnóstico educativo, Educación Media Superior.

\begin{abstract}
The problem that arises in this document is the absence of Physical Education programs for the Upper Middle School in the State of Mexico. Before this circumstance, the need arises to construct a diagnosis that reflects the educational reality. Results and analysis of surveys applied to teachers and students of Upper Middle Education of the State of Mexico are presented. The information obtained served as core to elaborate an educational diagnosis of needs and expectations that determined the relevance and feasibility of programs for the subject of Physical Education, which have to be implemented in a first phase in the 2018-2019 school year.
\end{abstract}

\section{Keywords}

Physical education, educational diagnosis, Upper Middle School.

\section{Introducción}

Este documento presenta el diagnóstico sobre necesidades y expectativas de alumnos y docentes de Educación Media Superior en el Estado de México. La información es resultado del análisis de dos encuestas realizadas (alumnos y docentes) en el ciclo escolar 2017-2018, en ellas se presentan los datos relevantes que dieron pauta al diseño de los programas de Educación Física en este nivel.

La Escuela Normal de Educación Física "General Ignacio M. Beteta", en su calidad de formadora en expertos en el área, a petición de la Dirección de Educación Media Superior del Estado de México, realizó un estudio diagnóstico que dio cuenta de la realidad educativa, así como de las necesidades y expectativas de docentes y alumnos de este nivel, para determinar la factibilidad y construcción de los programas de educación física que han de implementarse en el Estado a partir del ciclo escolar 20182019.

El documento está estructurado en su primer apartado por el problema de estudio, en él, se plantea la ausencia de programas de educación física en Educación Media Superior; en seguida las preguntas que orientaron el estudio y el objetivo; el siguiente apartado aborda la metodología empleada; por último, se presentan los resultados y el marco legal que sustenta la propuesta.

Diagnóstico de necesidades educativas para la construcción de programas... 


\section{El problema de estudio}

Históricamente en el Estado de México, el Plan de Estudios de nivel Medio Superior no ha considerado a la educación física como una asignatura. En virtud de ello no han existido programas educativos que aborden contenidos relativos a la actividad física desde una postura didácticopedagógica, las actividades que se han desarrollado son prácticas no reguladas que son propuestas llevadas a cabo por los docentes desde sus propios referentes, que en su totalidad son empíricos dado que el número de profesionales en esta área y en este nivel son escasos.

A partir de 1992 se incorpora de manera regular la actividad física en este nivel. Estas se llevaban a cabo como una propuesta cocurricular ya que no estaba considerada como asignatura en el mapa curricular, es decir, se trataba de actividades que eran no obligatorias, eso generaba que los planteles tenían la opción de realizarlas o no, en tanto que se carecía de programas específicos.

El documento que regula la actividad física en este nivel a partir de 2010 en el Estado de México, es un material propuesto por la Secretaría de Educación Pública (SEP), " Lineamientos de actividades físicas, deportivas y recreativas", en éste, se proponen actividades con relación al trabajo para desarrollar capacidades físicas, deportivas y de cuidado de la salud. El propósito es contribuir en la formación integral del alumno, de tal modo que constituye solo un apoyo a los responsables de este tipo de actividades en las instituciones que lo consideran pertinente.

El documento antes referido aporta solamente lineamientos de trabajo muy generales. Esta condición genera incertidumbre en los docentes que imparten esta asignatura cocurricular dado que no son especialistas del área, en su mayoría son docentes habilitados, es decir, tienen una formación distinta.

De lo anterior se considera indispensable el diseño de programas educativos para este nivel en la asignatura de Educación Física. En el Estado de México habrá de implementarse en el ciclo 2018-2019 los programas de Educación Física para educación Media Superior; éstos se construyeron a partir de un estudio diagnóstico que valoró su pertinencia y factibilidad.

\section{Preguntas y objetivos}

Para la realización del diagnóstico se consideraron los puntos de vista y sentidos que le otorgan los principales actores de los procesos educativos (alumnos y docentes) en Educación Media Superior, acerca de sus necesidades y expectativas relativas a los posibles contenidos de las 
RECIE. Revista Electrónica Científica de Investigación Educativa Vol. 4, núm. 2, enero-diciembre 2019, pp. 1049-1057.

asignatura en cuatro semestres. Estas se orientaron con las siguientes preguntas básicas:

1. ¿Qué tipo de actividades consideran alumnos y docentes debe trabajarse en la sesión de Educación Física?

2. ¿Qué contenidos consideran alumnos y docentes necesarios de incluir en el programa de Educación Física?

El objetivo de la construcción del diagnóstico fue aportar información relevante para el diseño de los programas de la asignatura de Educación Física en Educación Media Superior.

\section{Metodología}

Para la construcción de los programas de asignatura de educación física en educación Media Superior se consideró un diagnóstico. Se basó dos encuestas: una dirigida a profesores de Educación Media Superior y otra a alumnos que cursan el bachillerato. La información que se obtuvo puso en evidencia las necesidades y expectativas de los docentes y estudiantes, el punto de énfasis fue lo relacionado con la educación física en el Estado de México; las encuestas tomaron una muestra de 3468 profesores y 9018 alumnos durante el ciclo escolar 2017-2018.

En las encuestas se consideraron aspectos prioritarios que implican esta propuesta tales como: la corporeidad, iniciación deportiva, deportes, cultura física y salud. Se tomaron en cuenta las diferentes regiones del Estado de México y sus municipios, los ámbitos poblacionales de media superior, para advertir las necesidades que justifican esta propuesta curricular.

El análisis de la información que se obtuvo de las encuestas se realizó en dos momentos: el primero de ellos en relación al análisis y clasificación de las repuestas de los alumnos, en el segundo se realizó el mismo proceso con las respuestas de los docentes. A partir de ello se contrastó la información para determinar tendencias relacionadas con las actividades y contenidos, para posteriormente considerar su inclusión y progresión en cada uno de los semestres.

\section{Resultados y discusión}

En el Estado de México, se oferta el bachillerato en dos modalidades: general y tecnológico. Existen 523 planteles de bachillerato general y tiene un cobertura en 112 municipios con una matrícula de estudiantes de 218 661, una planta docente de 12299 y 54 supervisores. En el bachillerato tecnológico son 192 planteles, la cobertura del servicio está presente en 93 
municipios la matrícula de estudiantes asciende a 73 991, la planta docente es de 6282 y en esta modalidad son 26 supervisores.

Con relación a las respuestas de los alumnos: de un total de 9023 , el $80.4 \%$ cursan bachillerato general y el $19.6 \%$ el tecnológico. La tendencia está orientada al bachillerato general, se puede deducir que un porcentaje importante de alumnos aspiran a cursar estudios posteriores a este nivel, es decir, una licenciatura; por tanto, hay la necesidad que desarrollen competencias orientadas a un estilo de vida activo y saludable.

El sexo de los alumnos en la muestra que se consideró para este diagnóstico, el $51.9 \%$ son mujeres y el $48.1 \%$ son hombres. De acuerdo con datos del INEGI (2010) y II conteo de población y vivienda (INEGI, 2005), la tendencia poblacional esta edad es similar, a partir de estas cifras puede percibirse la necesidad de fomentar una educación incluyente y con equidad.

Las edades de los alumnos que integraron la muestra se clasificaron de la siguiente manera: el $41.5 \%$ se encuentra entre 14 y 15 años, el $50.7 \%$ se encuentra entre 16 y 17 años y $7.8 \%$ entre 18 y 19 años; se hace evidente la congruencia entre las edades de los alumnos con el grado que cursan, un escaso porcentaje son alumnos que por alguna razón se encuentran rezagados. La consistencia en las edades de los alumnos posibilita el diseño de actividades motrices, acordes a la etapa de desarrollo.

Con relación al tipo de actividades que los alumnos que integraron la muestra consideran que deberían estar en los programas de la asignatura son: $35.8 \%$ sugieren deportes (fútbol, basquetbol, voleibol y atletismo); $31.6 \%$ se inclinan por actividades recreativas, deportes, capacidades físicas; el $23.9 \%$ sugiere actividades recreativas (campamentos, caminatas ecológicas, etc.); el $19.4 \%$ desarrollo de capacidades físicas (fuerza, flexibilidad, resistencia y velocidad) y el $5.3 \%$ aspectos de salud.

Estos datos ponen en evidencia el tipo de actividades y contenidos que los alumnos de educación media superior sugieren incorporar en la propuesta curricular. Así mismo, sirven como elementos importantes a tomar en cuenta en los contenidos programáticos que se han de considerar en la construcción de los programas de asignatura en cada uno de los semestres.

Respecto de temas relacionados con el cuidado de la salud, los alumnos se pronunciaron de la siguiente manera: el $44.5 \%$ señaló la práctica de hábitos alimenticios saludables; el $31.5 \%$ propone la actividad física (caminata, bicicleta, danza, gimnasia, etc.) como contenidos que debieran estar presentes en el programa; en este mismo sentido se pronuncia el $27.9 \%$ sobre deportes de alto rendimiento (fútbol, basquetbol, 
RECIE. Revista Electrónica Científica de Investigación Educativa Vol. 4, núm. 2, enero-diciembre 2019, pp. 1049-1057.

voleibol, atletismo) y el $14.7 \%$ propone el juego motor (actividades físicas lúdicas) como contenidos importantes a considerar en los programas de la asignatura.

La información obtenida sirvió como base para la construcción de esta propuesta curricular. Con ella se pretende dar respuesta a las necesidades reales de los alumnos respecto de los contenidos necesarios a considerarse en la estructura de los programas de la asignatura de educación física en Educación Media Superior.

Se consideró también la opinión de los docentes que laboran en este nivel educativo. Información relevante en torno a tipo de bachillerato, antigüedad en el servicio, perfil profesional, elementos que consideran importantes para planear las sesiones y contenidos relevantes para ser considerados en esta propuesta.

Del cien por ciento de la muestra que laboran en Educación Media Superior, se clasificó a la población de la siguiente manera. De un total de 3 468 respuestas el $81.2 \%$ se desempeñan en bachillerato general, y el 18.8\% laboran bachillerato tecnológico. Como se puede observar, las posibilidades laborales están centradas en el bachillerato general dado que hay mayor demanda debido a la población existente en esta modalidad.

Con relación a la antigüedad en el servicio, los profesores participantes en el diagnóstico tienen diferentes rangos: de 1 a 5 años 842 docentes, éstos representan el $24.4 \%$; de 6 a 10 años 731 docentes, equivalente al $21 \%$ de la muestra; de 11 a 15 años 483, esto es el $13.9 \%$ y; más de 15 de servicio 1414 docentes, este número constituye el $40.7 \%$ del total de la muestra. De acuerdo con estos datos el $59.3 \%$ de los docentes que laboran en Educación Media Superior tienen menos de 15 años de servicio, se considera cuentan con la experiencia suficiente para identificar las necesidades actuales de formación de los alumnos que cursan este nivel. El perfil profesional de los docentes que laboran en Educación Media Superior es muy amplio. Involucra diferentes áreas de especialidad; psicología, derecho, pedagogía, ingeniería, ciencias sociales, computación, contaduría, etc. Estos datos son relevantes, se deben a la atención de la malla curricular de este nivel; un dato revelador es la cantidad reducida de educadores físicos (21 docentes) insertos en este nivel. Esta condición pone de manifiesto dos aspectos: el primero refiere un área de oportunidad para los docentes de Educación Física; el segundo, una necesidad apremiante de especialización o actualización de los docentes que atienden esta asignatura. 
Los documentos que los docentes consideran necesarios para la planificación de sesiones de Educación Física en este nivel, el $72 \%$ son los programas. En este sentido, se advierte la necesidad de que los maestros cuenten con programas de la asignatura como elemento esencial para abordar los contenidos, dado que la mayoría de ellos no son especialistas en el área. El 15.9 \% considera el documento referente al Modelo Educativo 2017, como principal referente para la planificación, y el $8 \%$ refiere la necesidad de emplear bibliografía especializada, el $5 \%$ manifestó falta de claridad al respecto.

Con relación al tipo de actividades que consideran deben realizarse en las sesiones de Educación Física. El $13.4 \%$ propone actividades recreativas (campamentos, caminatas, acantonamientos, etc.), el $17.8 \%$ sugiere ejercicio físico bajo la conducción de un docente, el $23.7 \%$ se pronunció por los deportes (futbol, básquetbol, atletismo, voleibol, etc.) y el $29.1 \%$ considera necesario el desarrollo de capacidades físicas (fuerza, velocidad, resistencia y flexibilidad) y el $53.3 \%$ piensa que todas las anteriores son importantes de realizarse. Estos datos sirvieron de base para estructurar las propuestas de contenidos en la asignatura de educación física.

Sobre los contenidos opinan que la alimentación y nutrición son temas importantes que deben estar presentes en los programas de Educación Física. Del mismo modo temas relacionados con la higiene, la obesidad y la sexualidad. Estas temáticas refieren problemas actuales de los jóvenes que cursan el bachillerato, por ello requieren ser abordados desde la prevención y la atención.

También proponen el desarrollo de habilidades físico deportivas, motricidad, corporeidad, juegos autóctonos, torneos deportivos, acondicionamiento físico. Estas propuestas de contenidos aportaron información relevante acerca de la percepción de los docentes con respecto a aquellos elementos que por su importancia debieran ser incorporados a los programas de la asignatura.

Este diagnóstico permitió la posibilidad de dar contenido a los cuatro programas de Educación Física de Bachillerato. Estos consideran las aportaciones tanto de estudiantes como de docentes, con base en ellas se plantean las propuestas que han de trabajarse en cada uno de los semestres. Con base en los resultados obtenidos en el diagnóstico se pone en evidencia la pertinencia para la implementación de los programas de atención al cuerpo y la salud en Educación Media Superior, la cual es normada en distintos documentos de carácter nacional e internacional. 
La política internacional expresada en la Declaración de Berlín (UNESCO, 2013) en la Conferencia Internacional de Ministros encargados del Deporte, considera la educación física en la escuela y en todas las demás instituciones educativas es el medio más efectivo para dotar a todos los niños y jóvenes de competencias, aptitudes, actitudes, valores, conocimientos y comprensión para su participación en la sociedad a lo largo de la vida , ahí se señalan los principios que han de prevalecer en la Educación Obligatoria y deberán atender las instituciones que ofrezcan estudios de Educación Media Superior.

La Carta Internacional de la educación física, la actividad física y el deporte (UNESCO, 2015) considera en su artículo primero que la práctica de la educación física, la actividad física y el deporte es un derecho fundamental para todos; en su artículo segundo; que la educación física, la actividad física y el deporte pueden reportar una amplia gama de beneficios a las personas, las comunidades y la sociedad en general.

En su artículo cuarto, que los programas de educación física, actividad física y deporte han de concebirse de tal modo que respondan a las necesidades y características personales de quienes practican esas actividades a lo largo de toda su vida; y en el siete señala que todo sistema educativo debe asignar el lugar y la importancia debidos a la educación física, la actividad física y el deporte, con miras a establecer un equilibrio y fortalecer los vínculos entre las actividades físicas y otros componentes de la educación; que el deporte y la educación física en la escuela y en todas la demás instituciones educativas formen parte integrante de las actividades cotidianas de los niños y los jóvenes.

El Estado Mexicano genera decisiones que corresponden con las tendencias mundiales en principios que están presentes en la Constitución Política de los Estados Unidos Mexicanos de 1917 que en su Artículo 3o, plantea:

- La educación será democrática, considerando a la democracia no solamente como una estructura jurídica y un régimen político, sino como un sistema de vida fundado en el constante mejoramiento económico, social y cultural del pueblo.

- $\quad$ Será de calidad, con base en el mejoramiento constante y el máximo logro académico de los educandos. 
El Plan Nacional de Desarrollo 2013-2018 (Poder Ejecutivo Federal, 2017), reconoce que las actividades físicas y deportivas favorecen la convivencia, la cooperación, la disciplina y otros valores que forman parte del currículo no académico que se ofrece a los estudiantes para una formación integral. Propone fomentar que la población escolar tenga acceso a la práctica de actividades físicas y deportivas en instalaciones adecuadas, con la asesoría de personal capacitado.

En este sentido el Programa Sectorial de Educación 2013-2018 (Diario Oficial de la Federación , 2013) hace mención específica acerca de su promoción en la educación media superior, para que los estudiantes cuenten con una infraestructura adecuada y de programas que la hagan posible en cada institución educativa.

La importancia de este estudio radica en que sirvió de base para el diseño de programas educativos de educación física en Educación Media Superior. Se aportaron datos de referentes empíricos que dieron cuenta de las necesidades y expectativas de docentes y alumnos con relación a actividades y contenidos que deberán ser abordados en la asignatura.

\section{Referencias}

Constitución Política de los Estados Unidos Mexicanos. (5 de Febrero de 1917). Camara de Diputados. Recuperado el 12 de abril de 2018, de Diario Oficial de la Federación: http://www.diputados.gob.mx/LeyesBiblio/pdf/1_150917.pdf Diario Oficial de la Federación . (13 de diciembre de 2013). Programa Sectorial de Educación 2013-2018. Recuperado el 13 de abril de 2018, de http://www.dof.gob.mx/nota_detalle_popup.php?codigo=5326569

INEGI. (2005). II conteo de población y vivienda. Recuperado el 13 de abril de 2018, de http://www.beta.inegi.org.mx/proyectos/ccpv/2005/

INEGI. (2010). El Censo General de Población y Vivienda del 2010.

Recuperado el 13 de abril de 2018, de http://www.beta.inegi.org.mx/proyectos/ccpv/2010/

Poder Ejecutivo Federal. (2017). Plan Nacional de Desarrollo 2013-2018.

Recuperado el 11 de abril de 2018, de http://pnd.gob.mx/ UNESCO. (2013). Conferencia Internacional de Ministros encargados del Deporte de la UNESCO. Recuperado el 12 de abril de 2018, de https://www.gob.mx/cms/uploads/attachment/file/128121/MINE PS_V_Informe_final.pdf

UNESCO. (2015). UNESCO.org. Recuperado el 11 de abril de 2018, de http://unesdoc.unesco.org/images/0023/002354/235409s.pdf

Peimbert Angulo, C.R.; Castaño Enríquez, C; y Meza Espinoza, H. 
RECIE. Revista Electrónica Científica de Investigación Educativa Vol. 4, núm. 2, enero-diciembre 2019, pp. 1049-1057. 